\title{
Rehabilitation of the Upper Extremity after Stroke: A Case Series Evaluating REO Therapy and an Auditory Sensor Feedback for Trunk Control
}

\author{
G. Thielman ${ }^{1}$ and P. Bonsall ${ }^{2}$ \\ ${ }^{1}$ Department of Physical Therapy, University of the Sciences, Philadelphia, PA 19104-4495, USA \\ ${ }^{2}$ Department of Occupational Therapy, Magee Rehabilitation Hospital, Philadelphia, PA 19106, USA
}

Correspondence should be addressed to G. Thielman, g.thielm@usp.edu

Received 13 April 2012; Revised 11 June 2012; Accepted 17 June 2012

Academic Editor: Marco Iosa

Copyright ( 2012 G. Thielman and P. Bonsall. This is an open access article distributed under the Creative Commons Attribution License, which permits unrestricted use, distribution, and reproduction in any medium, provided the original work is properly cited.

\begin{abstract}
Background and Purpose. Training in the virtual environment in post stroke rehab is being established as a new approach for neurorehabilitation, specifically, ReoTherapy (REO) a robot-assisted virtual training device. Trunk stabilization strapping has been part of the concept with this device, and literature is lacking to support this for long-term functional changes with individuals after stroke. The purpose of this case series was to measure the feasibility of auditory trunk sensor feedback during REO therapy, in moderate to severely impaired individuals after stroke. Case Description. Using an open label crossover comparison design, 3 chronic stroke subjects were trained for 12 sessions over six weeks on either the REO or the control condition of task related training (TRT); after a washout period of 4 weeks; the alternative therapy was given. Outcomes. With both interventions, clinically relevant improvements were found for measures of body function and structure, as well as for activity, for two participants. Providing auditory feedback during REO training for trunk control was found to be feasible. Discussion. The degree of changes evident varied per protocol and may be due to the appropriateness of the technique chosen, as well as based on patients impaired arm motor control.
\end{abstract}

\section{Background and Purpose}

Stroke is the leading cause of long-term disability in the United States, as there are estimated 6.4 million Americans that have survived a stroke and are now living with minor-tosevere activity limitations [1]. Activity limitations are often associated with persistent impairment of the upper limb. Specific impairments in this group can range from loss of range of motion, to impaired force generation, to decreased reaction times. These impairments can all lead to loss of arm function and further deficits in motor control, severely affecting a person's ability to live independently [2].

Training utilizing repetitive task practice strategies in poststroke rehab is an evolving method of treatment with a variety of treatment regimens possible. Recently, the application of various robotic systems to incorporate repetitive task practice and facilitate real-world tasks in stroke rehabilitation has been investigated [3-6]. However, since this groundbreaking form of treatment is so new, protocols are not well established and the differences between the robotic devices being used have led to difficulty in establishing specificity within protocols.

In 2 recent reviews evaluating the effect of robotic therapy on the upper limb of individuals after stroke, the high variability of all the work completed to date limited the determination of the effectiveness of this type of therapy $[3,4]$. One report indicated that even with robotic training, compensatory movement strategies were still a confluence and needed to be controlled [3]. According to prior robotic therapy investigations in this realm, the ideal candidates are those individuals who are quite impaired in their movement and are unable to perform the forward reaching movement without having the arm supported from the elbow to the hand [3-6]. However, individuals after stroke in the acute 
phase [5] as well as in the chronic phase [5] with mild-tomoderately severe impairments, have been shown to benefit from robotic therapy through various measures.

One delving question in poststroke rehabilitation is what is beneficial with individuals who are quite impaired and how great is the level of impairment where one can still benefit from the therapy [6]? One investigation that attempted to answer this question trained individuals at least 6 months after stroke onset, who were considered moderate to severe (average upper extremity Fugl Meyer \{FMA\} 9/66) and utilized passive supported therapy in a gravity-eliminated position [7]. The results for that investigation revealed impairment and disability level changes (functional changes were not assessed). One multicenter, randomized controlled trial evaluating the effectives of robotics in long-term upperlimb impairment led to no improvement in motor function immediately after training, but did show improved outcomes 9 months after therapy was complete (as compared to usual care with nondescript therapy) [8]. There was a third comparative group in that investigation that led to similar changes as the robotic group. Thus, more intense analysis of robotic therapy is necessary to help us understand how these results can translate into regularly used training protocols.

The robot-aided device REO has had only one pilot investigation and one feasibility study completed to date $[9,10]$. This REO system involves the patient being securely constrained to a device that can facilitate passive, active assisted, and active movement while avoiding compensatory trunk movement. Similar to the robotic devices discussed previously, the REO provides visual and auditory feedback as a means to enhance motor learning. In addition, this device forces the restriction of the trunk movement by use of a strap across the shoulder connected to the chair. The one pilot investigation mentioned did result in changes for a variety of patient levels on various outcome measures (FMA, strength, spasticity, and pain); however, no control condition was utilized [9]. The feasibility investigation noted that moderately impaired individuals after stroke (FMA 35/66) that were in inpatient rehab were accepting of the device and demonstrated significant changes on the FMA, but again there was no control condition [10].

Several investigations that have controlled the compensatory strategies of individuals after stroke onset have been undertaken recently [11-13]. A review paper [11] and a proof of concept paper [12] evaluated how controlling compensatory movements through feedback was incorporated into upper-limb recovery. The limited randomized controlled investigations that were found in that review, suggested that individuals who have had a stroke benefit from performance feedback in the physical environment (such as motor control deficits involving the controlling of trunk movement) to preserve motor learning abilities [11]. In other words, if the individuals can learn to control their trunk movements, motor recovery of the upper limb as evidenced by kinematic changes, can be made. The proof of concept paper noted clinical improvements on the FMA in more severely impaired poststroke individuals, when given proprioceptive feedback about the involved arm [12]. Thus, incorporating controlling trunk and/or arm movements has been shown to be an effective training method for individuals at various levels of impairment after stroke.

Systematic reviews of task-specific training have indicated that intensity and task specificity are primary indicators of effective treatment for individuals after stroke $[3,14]$. Upper-limb training investigations for individuals who have suffered a stroke have shown improved arm movement ability after completing the task-related training (TRT) with the impaired upper limb [15-17]. One particular method investigated showed that restricting trunk use, as is also used with the REO, has some short-term functional benefits as well as benefits to recovery of the impaired arm (interjoint coordination via reorganization of the arm versus trunk degrees of freedom), but no functional benefits $[15,18]$. The concern reported is that the trunk stabilizer becomes a dependent force on which the patient relies to achieve greater arm movement. Additionally, the present protocols using the REO, as mentioned previously, have not been adapted to the recent findings addressing the minimal functional effects of training with the trunk strapped down. Lastly, the appropriate level of patient to most benefit from unrestrained TRT has previously been defined in the literature as moderately impaired on the FMA (scoring between 20 and 40), and TRT has been well tested out in terms of trunk stabilization feedback, thus making for a solid control condition $[15,17]$.

The present case series utilized TRT as a control condition in a crossover comparison design, to obtain preliminary data for establishing a REO protocol for the upper limb. The purpose of this case series was to measure changes that can be made by incorporating a novel form of feedback to control trunk movements and auditory trunk sensor feedback during robot assisted arm therapy in moderate to severely impaired individuals after stroke. It was expected that all subjects would exhibit having learned to reach with greater use of their arm/less use of their trunk, exhibiting less dependency than with the stabilizer feedback.

\section{Case Descriptions}

Individuals with the following were excluded: receptive aphasia, apraxia, or other significant cognitive deficits (determined by the primary investigator during interview question and answer, as well as during command following during the screening sessions). Additional exclusions were (a) sensory/perceptual or orthopedic problems that limit one's ability to reach at the table without sliding the arm on the tabletop (discerned during initial physical exam screening using the Motor Assessment Scale \{MAS\} [19], a simple classification of arm and hand movements using the involved upper extremity) and (b) any cardiac or pulmonary conditions that limited exercise (physician clearance). The MAS cutoff was at level 4 for upper arm and 1 for hand, allowing for scapular stability/mobility and some grasping activity.

Inclusion criteria: demonstrating severe and moderately severe arm movement impairments, defined between 20 and 44 on the upper-arm subsection of the FMA (a series of 
functional multitask arm/hand movements, out of a possible score of 66) [20]. Individuals scoring below this range have previously been determined to be unable to participate in unrestrained TRT, receiving no benefit from the training [17] and individuals scoring greater than 50 have been considered only mildly impaired [21]. Written approval was obtained from the subject's physician after individual's consent; prior to actual participation and after subjects signed the informed consent the patient's primary physician was sent a letter describing the investigation, their patient's involvement, and asking for medical clearance via a waiver.

Three individuals after stroke voluntarily chose to be evaluated for the investigation, were ultimately included, and were randomized as to which protocol was given initially. All 3 individuals had a goal to be able to use their impaired arm for activities of daily living (ADLs). Subject (1): 63-yearold male, 3 years after onset of left-sided cerebrovascular accident (parietal lobe/basal ganglia), right side (dominant) sensorimotor deficits; subject (2): 68-year-old male 18 months after left-sided parietal lobe CVA that led to right side (dominant) sensorimotor deficits, subject (3): 56-year-old male 16 months after onset of right-sided parietal lobe CVA led to left-sided (nondominant) sensorimotor weakness.

2.1. Design. The institutional review boards at both the University of the Sciences and Magee Rehabilitation Hospital approved the study. Using a crossover design, four weeks of real-world TRT was first completed for 2 subjects, and then 4 weeks of REO therapy was completed after a 3-4week washout period where no training was performed. The reverse sequence was utilized for the third subject; REO followed by a 3-4-week washout and 4-week real-world TRT. This clinical trial is registered with the NIH clinical trials registry as NCT000844870.

2.2. Testing. Several standardized outcome measures were collected no longer than five days before the start of each training and within five days after completion of each training period. Due to the proximity of the testing in between the crossover period/washout period the initial postesting data was utilized as the subsequent pretesting data. Subjects were advised to continue to use their "new found" movement as much as possible, just as they had been advised during the training timeframe. The rehabilitative effects were measured according to the International Classification of Functioning, Disability and Health model (ICF) [22]. Body structure and function typically include motor impairment, while activity is measured by daily task performance, and participation measured by life situations.

The FMA [20] as well as the Reaching Performance Scale (RPS-measure of trunk movement used in reaching) [21], were used to determine body function and structure changes of the arm and trunk, respectively. Clinical measures of elbow and shoulder active range of motion (AROM) were assessed using a goniometer [23] (shoulder flexion was measured in sitting against gravity, while elbow extension was measured with gravity eliminated; the patients participating were not all able to complete elbow motion against gravity), and grip strength was measured using a dynamometer [24]. The average or 3 trials were used for all clinical measures. The MAS [19], used in pretest screening, was again collected at posttest as an additional body function and structure measure. Wolf Motor Function Test (WMFT-total time score to complete a number of tasks with impaired upper extremity) [25] was used as an activity measure, as well as the Motor Activity Log (MAL-patient's perception of function related to certain arm activities) [26]. Lastly, cognitive changes via the minimental status exam (MMSE-23 or lower is indicative of cognitive impairment, with a max score of 30) were collected [27].

\subsection{Intervention. The rehabilitative sessions lasted $40-45$} minutes, 2-3 days a week for up to 6 weeks (12 sessions). Subjects were seated with trunk-motion free. Both protocols were arranged to train with a wireless sensor pad draped across the back of the chair, cueing the subjects to keep their back against the pad whenever the back came off of the chair (see Figure 1). Figure 1 displays the individual with their back off of the sensor pad, initiating the auditory signal. Intermittent feedback for both groups was arranged by use of a faded feedback protocol in which the first third of the training trials were without the feedback $20 \%$ of the time, the second third of trials were without feedback for $40 \%$ of the time, and the final third was without feedback for $60 \%$ of the time [28]. This feedback protocol has previously been utilized in a TRT investigation [17].

REO allowed patients to perform robot-assisted activities, which involved continuous reaching with the arm placed in the device (see Figure 2). Visual feedback from the video monitor yielded the perceived best path to complete each motion. This marked path was overlaid by the subject's actual path taken. Verbal and some tactile feedback by the therapist was given during initial REO training, as the auditory sensor device proved to add a higher dimension of concentration for controlling trunk movement. Although the REO device has a shoulder restraint to control trunk movement, the goal was to not utilize this restraint. The goal was to complete motions while avoiding compensatory trunk movement, during passive, active-assisted, and active movement.

Variable practice TRT involved reaching to contact or grasp objects variably placed on the workspace, requiring arm movements of different amplitudes across all quadrants of the tabletop [15-17]. Common objects were used that varied in size, shape, and weight (e.g., cups, mugs, writing and eating utensils). Training activities included, sliding the arm across the tabletop with objects in hand and reaching to grasp/transport objects. As training progressed, subjects were encouraged to increase speed. Subjects received an auditory signal for feedback when the back came off of the pad. Instructions given were to move at preferred speed, and as training progressed subjects were encouraged to increase speed. If a session lasted less than $45 \mathrm{~min}$, subsequent sessions employed more complex tasks. As REO incorporated some active-assisted and some passive movements the time spent in therapy was the best way to equate the sessions and therefore, REO required $10-20 \%$ more training trials per session. During each session, 180-220 movements 


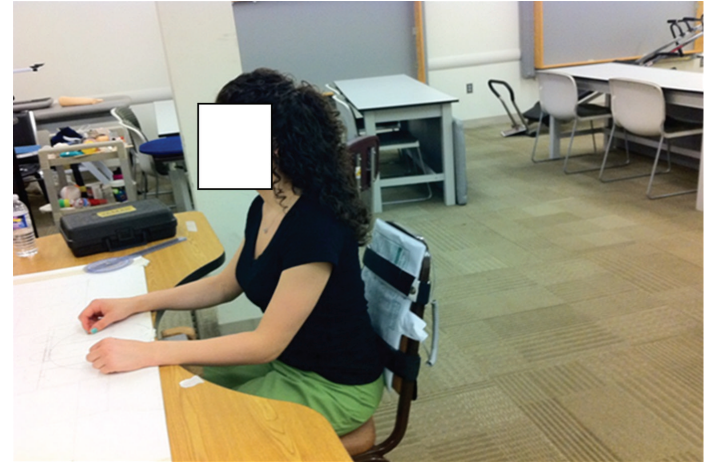

FIGURE 1: TRT workstation with sensor pad placed on anterior surface of chair back.

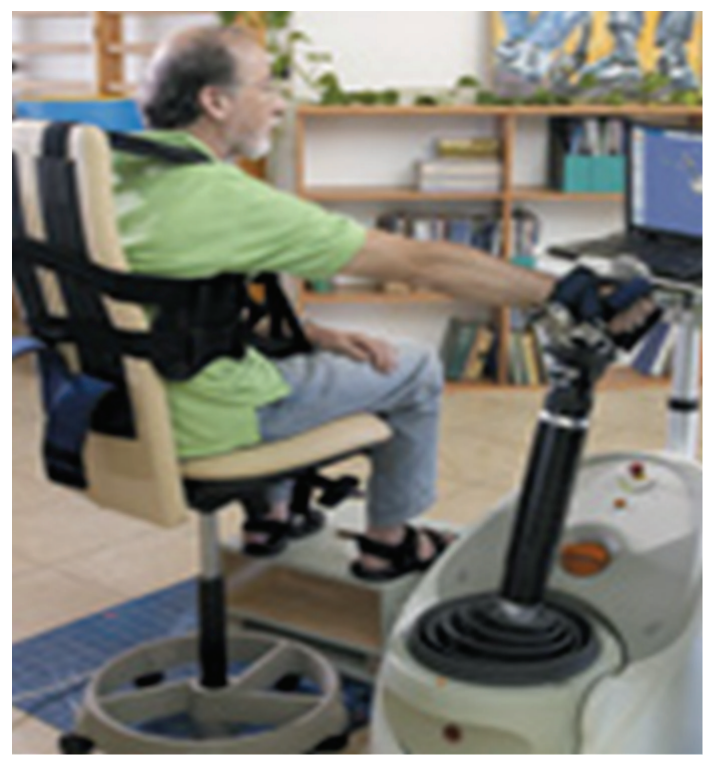

FIGURE 2: REO therapy training device (with trunk strap attached).

were performed for the TRT group, and 240-260 robotic movements (recording only movements reaching out away from the body in each protocol) were performed for the REO group, using the hemiparetic arm. Equivalent training time and feedback were controlled for with both protocols.

2.4. Analysis. Postests taken after each training bout, on each method, were completed and compared to pretraining scores. Significant changes were evident by relative change scores of $30 \%$ or greater. Based off of findings from other stroke investigations where minimal clinically important differences (MCID) after stroke revealed a $16 \%$ to $30 \%$ change as being meaningful for a number of the ICF measures we tested $[29,30]$, we conservatively used $30 \%$ for our results. A recent systematic review of outcome measures used for the evaluation of robot-assisted upper-limb exercise in stroke [31] has further details regarding the MCID for the FMA, grip strength, and WMFT, indicating a MCID of 7 for the FMA, $2.9 \mathrm{Kg}$ for grip strength, and 12 for the WMFT. We were only able to use the FMA and the grip strength since the WMFT score in their calculations was a calculated mean rate of performance score (functional ability scale), not the performance times as we used. We used the MAL as an equivalent measure to their mean rate of performance score.

\section{Results}

3.1. REO Performance. Subject 1 performed approximately the same amount of time on guided (passive) and initiated (active assisted) movement ( $40 \%$ each), with only $20 \%$ on the most difficult step-initiated (active) movement. Subject 2 performed about $30 \%$ of the time on the guided movement and $45 \%$ of the time on the initiated movement, and then $25 \%$ of the time on the most difficult step initiated activities. Subject 3 followed the same pattern as subject 1 for guided and initiated movement, but the percentages were $45 \%$ each therefore, leaving only $10 \%$ of the training on the most difficult step-initiated movement. The rationale for determining each of the programs and progress is a clinical decision based on biomechanics of the joint, capsular tightness, movement smoothness, and the ability to appropriately recruit the needed muscle pattern throughout the normal reaching pattern with no excessive compensatory mechanisms observed. The program progression is always to push the individual to the edges of their movement in which he or she is still able to maintain good reaching mechanics.

3.2. Outcome Measures. REO and TRT led to changes for all 3 participants (see Table 1). Table 1 notes the training order on the $x$-axis and the particular outcome measure on the $y$-axis, which are arranged according to ICF classification. Particular significant MCID training changes are highlighted for each subject.

Subject 1 displayed body function and structure level changes only after TRT for the MAS hand (65\% improvement), with no improvement apparent after the REO. Further body function and structure changes were indicated by the RPS (far and near), yielding 30\% improvement after REO, and this was maintained after TRT. Following this body function and structure level trend were elbow extension and grip strength (30\% improvement for each), both maintained after REO and TRT. Lastly, activity measure changes after REO were noted by the WMFT at $45 \%$ improvement, again maintained after TRT.

Subject 2 displayed activity level changes that were only apparent after TRT, indicated by the WMFT (45\% improvement). Subject 3 yielded body function and structure level changes after TRT that were maintained after REO $(30 \%$ improvement on RPS near and far). Additionally, subject 3 presented with activity level changes indicated by the WMFT, evident after TRT (35\% improvement) and maintained after REO. All other changes were below 30\% and were not considered to be as relevant as the findings reported here.

Observationally, the one initial body function and structure impairment that was limiting to a subject was the poor scapular stability, and capsular tightness/impingement of subject 3, exhibited by limited amount of shoulder flex and elbow extension; this permitted much less active 
TABLE 1: Outcome tests.

\begin{tabular}{|c|c|c|c|}
\hline & Subject 1 (REO first) & Subject 2 (TRT first) & Subject 3 (TRT first) \\
\hline \multirow{2}{*}{ Impairments pre-MAS- } & Upper Arm = 5/6 & Upper Arm = 5/6 & Upper Arm = 5/6 \\
\hline & Hand = 1/6 & Hand $=1 / 6$ & Hand $=6 / 6$ \\
\hline Posttraining 1/pretraining 2 & $5 / 6,2 / 6$ & $5 / 6,1 / 6$ & $5 / 6,6 / 6$ \\
\hline Posttraining 2 & 5/6, 5/6 (after TRT only)* & $5 / 6,1 / 6$ & $5 / 6,6 / 6$ \\
\hline Impairment pre-RPS(close) & $8 / 18$ & $8 / 18$ & $11 / 18$ \\
\hline Posttraining $1 /$ pretraining 2 & $13 / 18$ (after each)* & $9 / 18$ & $17 / 18$ (after each)* \\
\hline Posttraining 2 & $13 / 18$ (after each)* & $9 / 18$ & 16/18 (after each)* \\
\hline Impairments pre-RPS (far) & $8 / 18$ & $9 / 18$ & $11 / 18$ \\
\hline Posttraining $1 /$ pretraining 2 & $13 / 18$ (after each)* & $8 / 18$ & $17 / 18$ (after each)* \\
\hline Posttraining 2 & $13 / 18$ (after each)* & $10 / 18$ & $15 / 18$ (after each)* \\
\hline Impairments pre-FMA & $30 / 66$ & $26 / 66$ & $37 / 66$ \\
\hline Posttraining 1/pretraining 2 & $27 / 66$ & $26 / 66$ & $36 / 66$ \\
\hline Posttraining 2 & $27 / 66$ & $25 / 66$ & $40 / 66$ \\
\hline Impairment pre-shld. Flex & $98 \mathrm{deg}$ & 106 deg & $83 \mathrm{deg}$ \\
\hline Posttraining 1/pretraining 2 & $104 \mathrm{deg}$ & 133 deg & $89 \mathrm{deg}$ \\
\hline Posttraining 2 & $97 \mathrm{deg}$ & 114 deg & $90 \mathrm{deg}$ \\
\hline Impairment pre-elbow ext & $40 \mathrm{deg}$ & $66 \mathrm{deg}$ & $57 \mathrm{deg}$ \\
\hline Posttraining 1/pretraining 2 & 69 deg (after each)* & $63 \mathrm{deg}$ & $66 \mathrm{deg}$ \\
\hline Posttraining 2 & 71 deg (after each)* & $70 \mathrm{deg}$ & $64 \mathrm{deg}$ \\
\hline Impairment pre-grip str. & $12 \mathrm{lbs}$. & 5 lbs. & $10 \mathrm{lbs}$. \\
\hline Posttraining 1/pretraining 2 & 20 lbs. (after each)* & 5 lbs. & 12 lbs. \\
\hline Posttraining 2 & 21 lbs. (after each)* & 2 lbs. & $10 \mathrm{lbs}$. \\
\hline Activity pre-WMFT- & $216.94 \mathrm{sec}$ & $300.33 \mathrm{sec}$ & $179.24 \mathrm{sec}$ \\
\hline Posttraining 1/pretraining 2 & $112.97 \mathrm{sec}($ after each)* & $164.38 \mathrm{sec}$ (after TRT only) & $53.18 \mathrm{sec}(\text { after each })^{*}$ \\
\hline Posttraining 2 & $128.61 \mathrm{sec}(\text { after each })^{*}$ & $403.10 \mathrm{sec}$ & $49.76($ after each)* \\
\hline Activity pre-MAL & $5 / 70$ & $7 / 70$ & $18 / 70$ \\
\hline Posttraining 1/pretraining 2 & $7 / 70$ & $16 / 70$ & $30 / 70$ \\
\hline Posttraining 2 & $11 / 70$ & $14 / 70$ & $39 / 70$ \\
\hline Cognition pre-MMSE & $28 / 30$ & $29 / 30$ & $27 / 30$ \\
\hline Posttraining 1/pretraining 2 & $29 / 30$ & $29 / 30$ & $27 / 30$ \\
\hline Posttraining 2 & $29 / 30$ & $29 / 30$ & $28 / 30$ \\
\hline
\end{tabular}

MAS: Motor Assessment Scale, RPS: Reaching Performance Scale, FMA: Fugl Meyer Assessment, WMFT: Wolf Motor Function Test, MMSE: Minimental Status Exam, MAL: Motor Activity Log.

Asterisk after parentheses indicate at least 30\% change, after the particular therapy.

movement training (step initiated), as compared to the other 2 subjects. Conversely, subject 1 was especially able to utilize the REO for active movement, as his general scapular stability permitted extensive step-initiated movement.

\section{Discussion}

Both protocols used environmental feedback as a means to enhance motor learning. However, it became apparent during initial training that using the sensor to limit trunk movement during REO therapy was sensory overload for the subjects, and thus verbal and some tactile feedback from the therapist was necessary (at first) to control shoulder movement/compensatory movements of the trunk while completing the movement path outlined on the computer screen. The traditional method of forcibly restricting the trunk during REO has limited long-term functional use due to the poor carryover of trunk control once the restraint is removed, detracting from the ultimate goal of these protocols $[16,18,32,33]$. Conversely, using the REO with the trunk restraint can be very advantageous, allowing independent interaction with the REO, when clinicians are working on repetitive task practice and treating more than one patient at a time [34]. However, as the training therapist discovered during initial controlled training sessions, the modification in the training movement set by the REO device can also be used to limit the motion to a safe distance that does not cause any substitution.

Two out of three participants with moderate levels of body function and structure motor impairment were able to utilize a robotic therapy device, with these training modifications, to benefit the impaired upper limb. Particular REO performance guidelines can be gathered from this case series as all 3 subjects were trained with general training progression patterns, demonstrating that a moderately impaired 
patient would be able to perform at least 200 training movements reaching out away from one's body during the course of a typical training session. It was apparent that 10 $20 \%$ more arm movements per session with robotic therapy allowed for equivalent training session time, compared to more established TRT.

All 3 subjects made activity level changes as indicated by the WMFT, with subjects 1 and 3 maintaining these changes after each of the protocols. Subjects 1 and 3 also displayed body function and structure changes (RPS near and far), that were maintained after each of the protocols. Subject 2 made the least amount of changes, while no subject made changes on the other activity level measure (MAL), perhaps not being as sensitive as the WMFT.

As all 3 subjects did yield WMFT activity level changes, this is perhaps the best indicator of the benefits of these additional structured activities engaging their impaired arm in training. The RPS level changes for subjects 1 and 3 that were maintained for either therapy are a credit to the generalized focus of both types of therapy assisting in controlling arm versus trunk disassociation of movement. The focus of improving the problem of arm control in this population is clearly indicated with these impairments and addressable with the REO. Conversely, subject 2 made no changes as a result of the REO activities; in fact, the gains made on the WMFT and shoulder flexion were back to baseline after the completion of the training on the REO. The treating therapist felt that this was due to scapular immobility and capsular tightness. Thus, despite meeting the minimal criteria to be included in the investigation, the compensatory abilities may have initially masked the poor function of the scapula. Furthermore, subject 2 made no changes on the MAS hand score or with elbow extension. Thus, in comparison to the changes made by subjects 1 and 3 on these measures and in conjunction with the trunk control changes (RPS), we were able to confirm that compensatory trunk movement is used to assist arm reach, as well as hand orientation for grasping.

Clinically, the activity level change (MAL) represents a strong indicator of the patient's belief that they are benefiting from therapy, and with no subject perceiving this benefit, it may be a limitation of the measure. However, there were no changes on the FMA, which is often cited as a measure of motoric change and notably, both other published reports that utilized the REO demonstrated positive findings on the FMA as well as a high level of patient satisfaction $[8,9]$. Evaluating the changes that subject 1 made on the MAS hand section that were not seen on the FMA score is perhaps due to the fact that the body function and structure changes made were at the elbow, arm control (RPS), and grip strength, not on some of the finer hand and wrist activities tested out by the FMA.

The result that this auditory feedback training modification is not just possible with TRT but also with REO for this level of patient is promising and warrants further investigative evaluation. The benefits that are carried over from the initial training protocol to the next can be seen as a limitation, as it is unknown with such a short washout period if the overall benefit is the cumulative effect of additional therapy or the true effect of the actual therapy just received. Conversely, the progression of REO to TRT as a training protocol may be the ideal protocol for a patient with the level of impairments as subject 1 , as this was the most number of changes evidenced.

The use of Kinematic analysis in future investigations should lead to a differentiation of upper arm, lower arm movement, and trunk control as well as assist in determining control strategies that are developed as a result of training. A randomized controlled investigation with a population of subjects with similar deficits and perhaps similar time after stroke (as well as subsequent similar baseline measurements) would better clarify what impairments are better trained with REO, what impairments are better trained with more established protocols such as TRT, and the appropriate length of training of each.

\section{Conflict of Interests}

The authors declare there are no competing interests in this paper.

\section{Acknowledgments}

This paper was presented in part at the 2010 Combined Sections Meeting of the American Physical Therapy Association, San Diego, CA. Project funding is provided by USciences Office of Academic Affairs. Nicole Walsh, OTR/L assisted with the REO training at Magee Rehabilitation Hospital and the following USP Graduate Students assisted in writing the methodology, and performing the training on the subjects: Greg Oomen and Dave Sen.

\section{References}

[1] A. Tennant, J. M. L. Geddes, J. Fear, M. Hillman, and M. A. Chamberlain, "Outcome following stroke," Disability and Rehabilitation, vol. 19, no. 7, pp. 278-284, 1997.

[2] S. J. Page, G. D. Fulk, and P. Boyne, "Clinically important differences for the Upper-extremity fugl-meyer scale in people with minimal to moderate impairment due to chronic stroke," Physical Therapy, vol. 92, no. 6, pp. 791-798, 2012.

[3] G. Kwakkel, B. J. Kollen, and H. I. Krebs, "Effects of robotassisted therapy on Upper limb recovery after stroke: a systematic review," Neurorehabilitation and Neural Repair, vol. 22, no. 2, pp. 111-121, 2008.

[4] J. Mehrholz, T. Platz, J. Kugler, and M. Pohl, "Electromechanical and robot-assisted arm training for improving arm function and activities of daily living after stroke," Stroke, vol. 40, no. 5, pp. e392-e393, 2009.

[5] S. Masiero, A. Celia, G. Rosati, and M. Armani, "Roboticassisted rehabilitation of the Upper limb after acute stroke," Archives of Physical Medicine and Rehabilitation, vol. 88, no. 2, pp. 142-149, 2007.

[6] G. Morone, M. Iosa, M. Bragoni et al., "Who may have durable benefit from robotic gait training? A 2 year follow up randomized controlled trial in patients with subacute stroke," Stroke, vol. 43, no. 4, pp. 1140-1142, 2012.

[7] L. R. MacClellan, D. D. Bradham, J. Whitall et al., "Robotic Upper-limb neurorehabilitation in chronic stroke patients," 
Journal of Rehabilitation Research \& Development, vol. 42, no. 6, pp. 717-722, 2005.

[8] A. C. Lo, P. D. Guarino, L. G. Richards et al., "Robot-assisted therapy for long-term Upper-limb impairment after stroke," The New England Journal of Medicine, vol. 362, no. 19, pp. 1772-1783, 2010.

[9] F. Bovolenta, M. Goldoni, P. Clerici, M. Agosti, and M. Franceschini, "Robot therapy for functional recovery of the Upper limbs: a pilot study on patients after stroke," Journal of Rehabilitation Medicine, vol. 41, no. 12, pp. 971-975, 2009.

[10] I. Treger, S. Faran, and H. Ring, "Robot-assisted therapy for neuromuscular training of sub-acute stroke patients. A feasibility study," European Journal of Physical and Rehabilitation Medicine, vol. 44, no. 4, pp. 431-435, 2008.

[11] S. K. Subramanian, C. L. Massie, M. P. Malcolm, and M. F. Levin, "Does provision of extrinsic feedback result in improved motor learning in the Upper limb poststroke? A systematic review of the evidence," Neurorehabilitation and Neural Repair, vol. 24, no. 2, pp. 113-124, 2010.

[12] M. Casadio, P. Giannoni, P. Morasso, and V. Sanguineti, "A proof of concept study for the integration of robot therapy with physiotherapy in the treatment of stroke patients," Clinical Rehabilitation, vol. 23, no. 3, pp. 217-228, 2009.

[13] P. Lam, D. Hebert, J. Boger et al., "A haptic-robotic platform for Upper-limb reaching stroke therapy: preliminary design and evaluation results," Journal of NeuroEngineering and Rehabilitation, vol. 5, article 15, 2008.

[14] G. Kwakkel, B. Kollen, and E. Lindeman, "Understanding the pattern of functional recovery after stroke: facts and theories," Restorative Neurology and Neuroscience, vol. 22, no. 3-4, pp. 281-299, 2004.

[15] G. Thielman, T. Kaminski, and A. M. Gentile, "Rehabilitation of reaching after stroke: comparing 2 training protocols utilizing trunk restraint," Neurorehabilitation and Neural Repair, vol. 22, no. 6, pp. 697-705, 2008.

[16] G. Thielman, "Rehabilitation of reaching poststroke: a randomized pilot investigation of two types of trunk control," Journal of Neurologic Physical Therapy, vol. 34, no. 3, pp. 138$144,2010$.

[17] G. T. Thielman, C. M. Dean, and A. M. Gentile, "Rehabilitation of reaching after stroke: task-related training versus progressive resistive exercise," Archives of Physical Medicine and Rehabilitation, vol. 85, no. 10, pp. 1613-1618, 2004.

[18] S. M. Michaelsen, R. Dannenbaum, and M. F. Levin, "Taskspecific training with trunk restraint on arm recovery in stroke: randomized control trial," Stroke, vol. 37, no. 1, pp. 186-192, 2006.

[19] J. H. Carr, R. B. Shepherd, L. Nordholm, and D. Lynne, "Investigation of a new motor assessment scale for stroke patients," Physical Therapy, vol. 65, no. 2, pp. 175-180, 1985.

[20] D. J. Gladstone, C. J. Danells, and S. E. Black, "The fugl-meyer assessment of motor recovery after stroke: a critical review of its measurement properties," Neurorehabilitation and Neural Repair, vol. 16, no. 3, pp. 232-240, 2002.

[21] S. M. Michaelsen, S. Jacobs, A. Roby-Brami, and M. F. Levin, "Compensation for distal impairments of grasping in adults with hemiparesis," Experimental Brain Research, vol. 157, no. 2, pp. 162-173, 2004.

[22] International Classification of Function in Disability and Health: ICF, World Health Organization, Geneva, Switzerland, 2001.

[23] C. Norkin and D. White, Measurement of Joint Motion: A Guide To Goniometry, F. A. Davis Company, Philadelphia, Pa, USA, 1995.
[24] J. E. Harris and J. J. Eng, "Paretic Upper-limb strength best explains arm activity in people with stroke," Physical Therapy, vol. 87, no. 1, pp. 88-97, 2007.

[25] S. L. Wolf, P. A. Catlin, M. Ellis, A. L. Archer, B. Morgan, and A. Piacentino, "Assessing Wolf motor function test as outcome measure for research in patients after stroke," Stroke, vol. 32, no. 7, pp. 1635-1639, 2001.

[26] S. W. Park, S. L. Wolf, S. Blanton, C. Winstein, and D. S. Nichols-Larsen, "The EXCITE trial: predicting a clinically meaningful motor activity log outcome," Neurorehabilitation and Neural Repair, vol. 22, no. 5, pp. 486-493, 2008.

[27] F. Özdemir, M. Birtane, R. Tabatabaei, G. Ekuklu, and S. Kokino, "Cognitive evaluation and functional outcome after stroke," American Journal of Physical Medicine \& Rehabilitation, vol. 80, no. 6, pp. 410-415, 2001.

[28] R. Schmidt and D. L. Lee, Motor Control and Learning: A Behavioral Emphasis, Human Kinetics Books, Champaign, Ill, USA, 2005.

[29] A. J. Vickers and D. G. Altman, "Analysing controlled trials with baseline and follow up measurements," BMJ, vol. 323, no. 7321, pp. 1123-1124, 2001.

[30] C. E. Lang, D. F. Edwards, R. L. Birkenmeier, and A. W. Dromerick, "Estimating minimal clinically important differences of Upper-extremity measures early after stroke," Archives of Physical Medicine and Rehabilitation, vol. 89, no. 9, pp. 16931700, 2008.

[31] M. Sivan, R. J. O’Connor, S. Makower, M. Levesley, and B. Bhakta, "Systematic review of outcome measures used in the evaluation of robot-assisted Upper limb exercise in stroke," Journal of Rehabilitation Medicine, vol. 43, no. 3, pp. 181-189, 2011.

[32] S. M. Michaelsen and M. F. Levin, "Short-term effects of practice with trunk restraint on reaching movements in patients with chronic stroke," Stroke, vol. 35, no. 8, pp. 19141919, 2004.

[33] S. M. Michaelsen, R. Dannenbaum, and M. F. Levin, “Taskspecific training with trunk restraint on arm recovery in stroke: randomized control trial," Stroke, vol. 37, no. 1, pp. 186-192, 2006.

[34] N. G. Kutner, R. Zhang, A. J. Butler, S. L. Wolf, and J. L. Alberts, "Quality-of-life change associated with roboticassisted therapy to improve hand motor function in patients with subacute stroke: a randomized clinical trial," Physical Therapy, vol. 90, no. 4, pp. 493-504, 2010. 


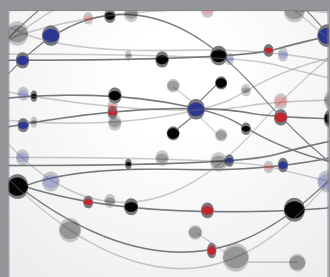

The Scientific World Journal
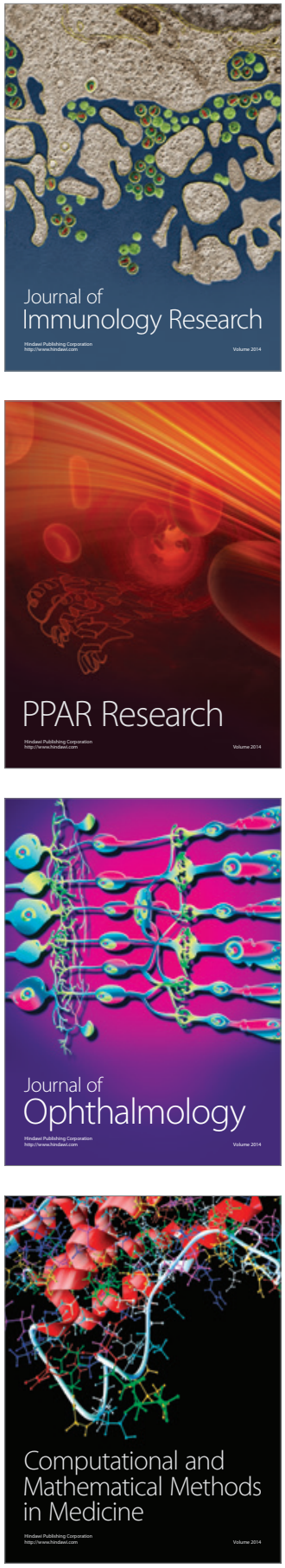

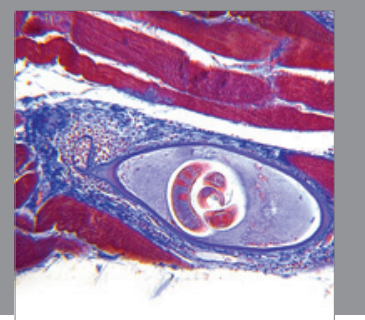

Gastroenterology

Research and Practice
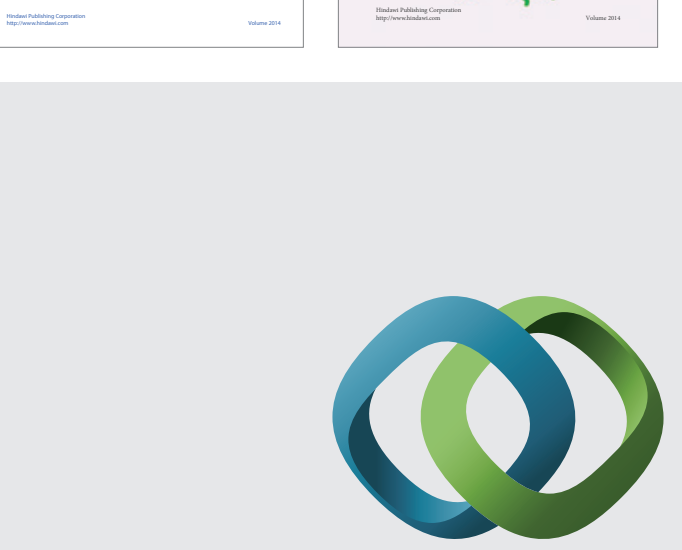

\section{Hindawi}

Submit your manuscripts at

http://www.hindawi.com
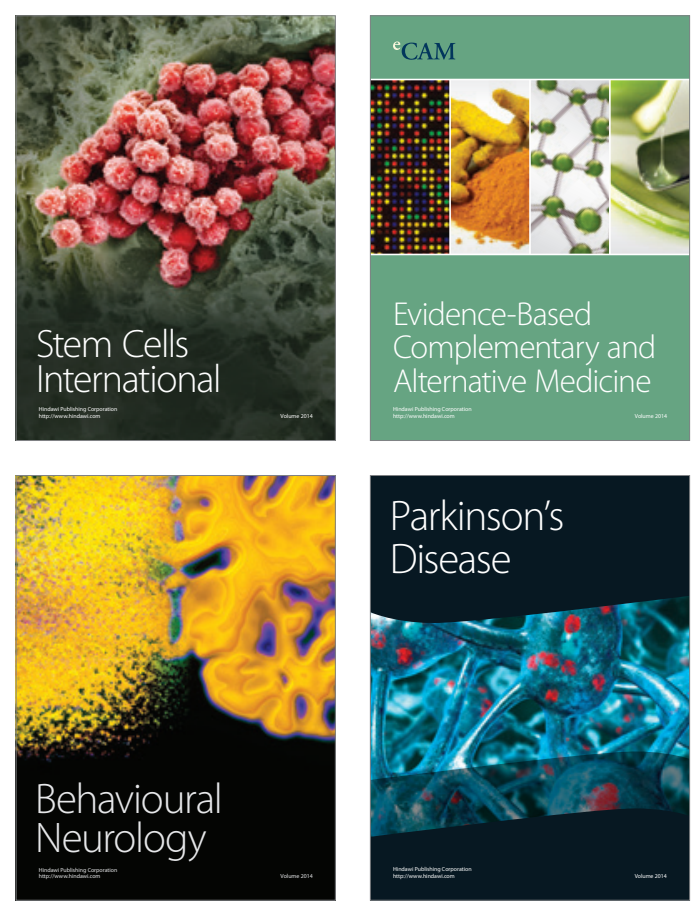

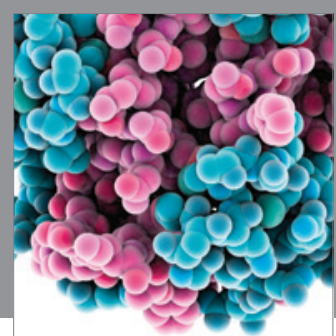

Journal of
Diabetes Research

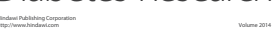

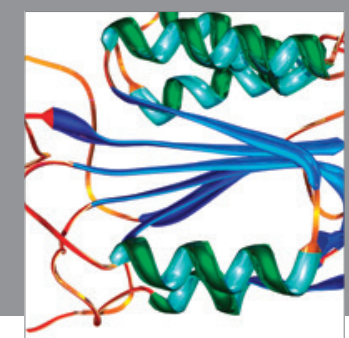

Disease Markers
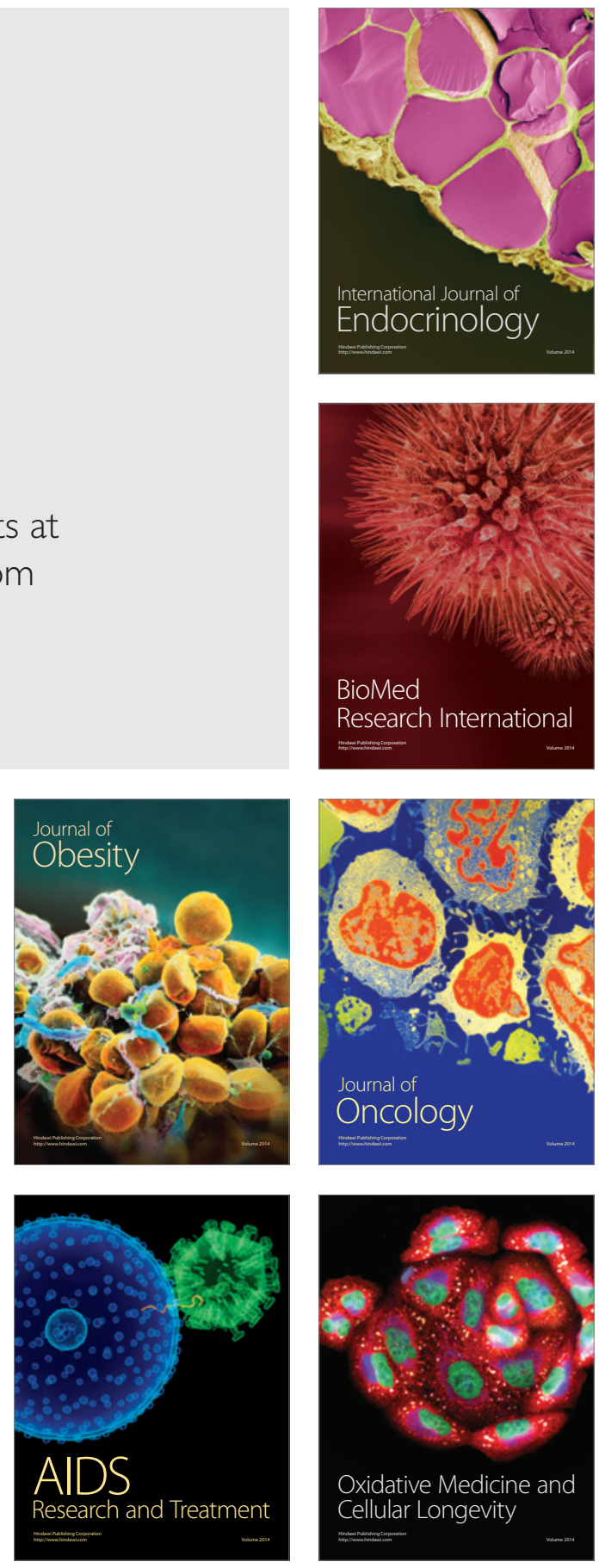